\title{
EFFECT OF RASA MEZHUGU ON FREUND'S ADJUVANT-INDUCED ARTHRITIS IN RATS
}

\author{
K. NANDHAGOPAL ${ }^{* 1}$, M. KANNIYAKUMARI ${ }^{2}$ \\ 1Department of Pharmacology, Sri Santhigiri Siddha Medical College, Trivandrum, Kerala 695589, India, 2Department of Pathology, Sri \\ Santhigiri Siddha Medical College, Trivandrum, Kerala 695589, India \\ Email: drnandhagopalsiddha@gmail.com
}

Received: 25 Jul 2016, Revised and Accepted: 17 Sep 2016

\begin{abstract}
Objective: Rheumatoid arthritis is a long-lasting autoimmune disorder that primarily affects joints. It typically results in warm, swollen, and painful joints. Pain and stiffness often worsen the following rest. The goal of treatment is to reduce pain, decrease inflammation, and improve a person's overall functioning. In spite of tremendous development in the field of synthetic drugs during recent years, the side effects could not be avoided. Hence the current research focus is to develop less toxic drugs as early as feasible in the disease process.
\end{abstract}

Methods: A 90 d old Wistar male rat was used for the research and was divided into four groups treated with Saline, Palm Jaggary, Rasa Mezhugu and Indomethacin.

Results: Acute toxicity study, Sub Acute study, Anti-arthritic study and histological findings showed that the Rasa Mezhugu has an anti-arthritis effect.

Conclusion: Further the histopathology slide photos of bone, cerebrum, cardiac muscle, kidney, liver, pancreas, ovaries, lungs, testes, stomach and spleen proved the safety of Rasa Mezhugu in rats.

Keywords: Rasa Mezhugu, Palm Jaggary, Indomethacin and Rheumatoid Arthritis

(C) 2016 The Authors. Published by Innovare Academic Sciences Pvt Ltd. This is an open access article under the CC BY license (http://creativecommons.org/licenses/by/4.0/)

DOI: http://dx.doi.org/10.22159/ijcpr.2016v8i4.15686

\section{INTRODUCTION}

Rheumatoid Arthritis (RA) is a chronic, destructive inflammatory polyarticular joint and systemic autoimmune disease of unknown cause. The prevalence of Rheumatoid Arthritis is consistent worldwide affecting, about $0.5-1.0 \%$ of the population. It usually occurs in people between 25 and 55 y of age. Women are affected more often than men at a ratio of 3 to 1 . It is characterized by synovial hyperplasia, angiogenesis and mononuclear infiltration. RA progresses in three stages. The stage-I is the swelling of the synovial lining, causing pain, warmth, stiffness, redness and swelling around the joint. Stage-II is the rapid division and growth of cells, or pannus, which causes the synovium to thicken. In stage-III, the inflamed cells release enzymes that may digest bone and cartilage, often causing the involved joint to lose its shape and alignment, more pain and loss of movement. As the result of the inherent problems associated with current non-steroidal as well as steroidal anti-inflammatory agents, there is a continuous search, especially from a natura source. The inflammatory response forms the basis of several pathological and pathophysiological processes including rheumatoid arthritis, atherosclerosis and neurodegenerative disorders [1,2].

The processes that underline the phenomenon of inflammation are now better understood, and so are the modes of action of drugs that are used to treat it. Acute and chronic inflammatory processes are a major threat to human health and play an important role in the development of various diseases. Acute inflammation is a rapid response to an injurious agent that serves to deliver mediators of host defense to the site of injury. Its main characteristics are the exudation of fluid and plasma proteins (oedema) and the emigration of leukocytes predominantly neutrophils. Acute inflammatory reactions are triggered by a variety of stimuli such as infections (Bacterial, viral, parasitic and microbial toxins); physical and chemical agents (burns, irradiation and environmental chemicals acute inflammatory reactions, in contrast to chronic inflammatory reactions, are usually self-limiting and resolve. Chronic inflammation occurs when acute inflammation remains unresolved. It is the reaction arising when the acute response is insufficient to eliminate the pro-inflammatory agents. It is of longer duration and is associated histologically with the presence of lymphocytes and macrophages, the proliferation of blood vessels, fibrosis and tissue necrosis. Chronic inflammation occurs in the following settings: Persistent infections by certain microorganisms (e. g., tubercle bacilli, virus, fungi, and parasites); prolonged exposure to potentially toxic agents and autoimmunity $[3,4]$.

Rheumatoid arthritis is caused by no of pro-inflammatory molecules released by macrophages including reactive oxygen species and eicosanoids such as prostaglandins, leukotrienes and cytokines. The regulation of these mediators secreted by macrophages and other immune cells and modulation of arachidonic acid metabolism by inhibiting enzymes like Cox and Lox are the potential target for chronic inflammatory conditions. Even though various categories like immunosuppressants, NSAIDs, steroidal anti-inflammatory drugs are being used till now, the potential side effects give a limitation for their use.

Complete Freund's adjuvant (CFA)-induced arthritis in rats is an inflammatory model widely used in etiopathogenic investigational drug and molecular studies due to its similarity to human rheumatoid arthritis [5].

The current research focus is to develop less toxic drugs as early as feasible in the disease process. In spite of tremendous development in the field of synthetic drugs during recent years, the side effects could not be avoided. Therefore, the efficacy of various plants against inflammation and arthritis has been explored. Now it is a growing concern all over for the development of new safe, potent, less toxic antiarthritic drug. Hence, there is a need to explore for more naturally available alternatives, so that their therapeutic values can be assessed and expanded.

\section{MATERIALS AND METHODS}

\section{Animals}

A $90 \mathrm{~d}$ old Wistar rat bred and raised in the Animal Facility of the Department of Pharmacology, Vel's college of Pharmacy was used in this study. They were maintained under constant automatically control $12 \mathrm{~h} / 12 \mathrm{~h}$ light/dark cycle (lights on from $07.00 \mathrm{a}$. m. to 07.00 p. m.) and environmental temperature $\left(23 \pm 2{ }^{\circ} \mathrm{C}\right)$. Rat chow 
and tap water were provided ad libitum in standard propylene cages. Cage cleaning consisted of a daily change of sawdust bedding. At the end of the study, animals were sacrificed with an overdose of Chloral hydrate. Due to the painful condition imposed on the animals, the number of subjects used was restricted to the minimum that allowed reliable statistical analysis of the results. All procedures were submitted to and approved by the Ethics Committee and followed the recommendations of the Research and Ethics Committee. Each group was composed of 6 animals $[6,7]$.

\section{Drug material and stock solution}

The semi-solid form of Rasa Mezhugu was mixed uniformly in Palm jaggary $(100 \mathrm{mgw} / \mathrm{v})$ in saline solution to achieve $100 \mathrm{mg} / \mathrm{ml}$ as main stock solution and used in this study. All solutions were daily prepared and stirred until the residues were completely dissolved. The volume administered was $2 \mathrm{ml} / \mathrm{kg}$ of body weight. Control animals received the same volume of Palm jaggary (100 mgw/v) in saline.

\section{Toxicity studies}

Evaluation of acute oral toxicity of Rasa Mezhugu was carried out according to the Acute Oral Toxicity-Up-and-Down Procedure by the Organization for Economic Co-operation and Development (OECD) guidelines for testing of chemicals (425). A limit test $(5000 \mathrm{mg} / \mathrm{kg}$ body weight) was performed using five male albino mice (28-33g) from our breeding stock. All the animals were observed for behavioral changes and mortality till $14 \mathrm{~d}$ after administration of the dose. Similarly, Evaluation of oral 28day toxicity of Rasa Mezhugu was carried out according to the Repeated Dose 28-day Oral Toxicity Study by OECD guidelines for testing of chemicals (407). Male Wistar rats (150-180 g) from our breeding stock were used for the subacute and anti-arthritic study. Drug/vehicle was administered daily for the duration of $28 \mathrm{~d}[8,9]$.

\section{Evaluation of anti-arthritic potency}

Freud's adjuvant-induced arthritis model was used to assess the anti-arthritic activity in albino rats. Following anesthesia with diethyl ether, $0.1 \mathrm{ml}$ of Freund's adjuvant (complete fraction of Mycobacterium butyricum suspended in mineral oil; was injected in the sub-plantar tissue of the right posterior paw and $100 \%$ of the animals developed arthritis. Every day animals were carefully and thoroughly inspected, by examining the affected paw and the animal's general status. Evaluation of the anti-arthritic effects of Rasa Mezhugu was performed by monitoring the edema in the right paw. In control animals, sub-plantar injection of Freund's adjuvant produced a local edema after a few hours with a progressive increase reaching its maximum in the eighth day after inoculation. On day 0 , all animals were subjected to behavioral test and assessment of body weight and right paw's measurements, followed by the injection of Freund's adjuvant in the right paw.

On the $7^{\text {th }}$ day after adjuvant administration, animals were randomly distributed into four groups: Group I received the vehicle saline only. Group II received the vehicle $(2 \mathrm{ml} / \mathrm{kg}$ body weight, Palm jaggary (100 mgw/v) in saline) and served as arthritic control and group III received Rasa Mezhugu with Palm jaggary at a dose of 500 $\mathrm{mg} / \mathrm{kg}$ body weight. Group-IV animals were treated with Indomethacin $(10 \mathrm{mg} / \mathrm{kg})$ and then again all those animals were subjected to behavioral test and assessment of body weight and paw's edema measurements. The test solution was administered daily, and the testing application was done on Days 8, 13, 18, 23 and 28 after injection of Freund's adjuvant. Paw volume was measured on $4^{\text {th }}, 8^{\text {th }}, 14^{\text {th }}$ and $21^{\text {st }}$ day with the help of Plethysmometer for both the ipsilateral (injected paw) and the contralateral paw (noninjected paw) before intraplantar injection of CFA (day 0) and every other day [10].

The volume of mercury displacement is correlated to the paw volume. Volume measurements were conducted twice for each paw, and the average volume displacement was calculated. The edema was quantified by measuring the difference in foot volume between day 0 and subsequent time points. The volumes measured on days post-injection were calculated as the percentage of the day 0 volume. The difference in the paw edema from the control group to that of treated group is taken as the anti-arthritic potential of Rasa Mezhugu.

The mean changes in injected paw edema with respect to initial paw volume were calculated on respective days and percentage inhibition of paw edema with respect to untreated group (control) was calculated using the formula: The percentage inhibition of edema for each group was calculated by using the following equation.

$$
\% \text { inhibition of edema }=\frac{\mathrm{N}-\mathrm{N}^{1}}{\mathrm{~N}} \times 100
$$

Where: $\mathrm{N}=$ Volume of edema in control; $\mathrm{N}^{1}=$ Volume of edema in treated group

\section{Arthritic index score}

\begin{tabular}{llc}
\hline Ears & Absence of nodules and redness & 0 \\
& Presence of nodules and redness & 1 \\
Nose & No swelling of connective tissue & 0 \\
& Intensive swelling of connective tissue & 1 \\
Tail & Absence of nodules & 0 \\
& Presence of nodules & 1 \\
Forepaws & Absence of inflammation & 0 \\
& Inflammation of at least 1 & 1 \\
Hind paws & Absence of inflammation & 0 \\
& Slight inflammation & 1 \\
& Moderate inflammation & 2 \\
& Marked inflammation & 3 \\
\hline
\end{tabular}

On $28^{\text {th }}$ day, all group animals were observed thoroughly for the above changes the score was calculated as per the above table. Normal-0, Control$1+1+1+1+3=7$, Rasa mezhugu- $0+0+0+0+1=1$, Standard- 0 . An arthritic index is calculated as the sum of the scores as indicated above for each animal. The average of the treated animals was compared with the control group.

\section{Histopathological assessment}

After euthanasia on $28^{\text {th }}$ day, the hind paws amputated above the knee joint and were fixed in $10 \%$ formalin solution. The paws were then decalcified using $10 \%$ Nitric acid embedded in paraffin and sectioned in a mid-sagittal plane. The sections of articulation of the tarsal joints were stained with haematoxylin and eosin and were examined microscopically for mononuclear infiltration, pannus formation and bone destruction. The hind paw was amputed above the knee joint and fixed in $10 \%$ formalin solution. The sections were stained with heamtoxylin and eosin and were examined microscopically for histopathological changes [11].

\section{Statistical analysis}

The statistical significance was assessed by using one-way analysis of variance (ANOVA) and followed by dunnet's comparisons test. All 
the data are presented as mean \pm SEM and $\mathrm{p}<0.05$ was considered as significant.

\section{RESULTS AND DISCUSSION}

\section{Acute toxicity study}

In acute toxicity studies, the Rasa Mezhugu up to $5000 \mathrm{mg} / \mathrm{kg}$ did not produce any toxic symptoms or mortality in mice, and hence the Rasa Mezhugu was considered to be safe and non-toxic, and one tenth of this maximum tolerable dose was used as the therapeutic dose for further pharmacological screening.

\section{Anti-arthritic study}

Adjuvant-induced arthritis in rats is a well-established experimental model that has features similar to the human rheumatoid arthritis. In addition, it is a good chronic inflammatory model for the development of potential analgesic and/or anti-inflammatory drugs useful for arthritis treatment. Adjuvant arthritis is characterized by chronic proliferative and inflammatory reactions in synovial membranes, producing pain, disability and eventually destruction of joints. Although, the etiology of this disease is unknown, it is thought that autoimmune processes are involved. Experimental evidence suggests that an autoimmune process involving $\mathrm{T}$ lymphocytes is responsible for the generation of adjuvant arthritis. In the present study, the challenge with CFA $(1 \%, 0.1 \mathrm{ml})$ was produced monoarticular arthritis, evident from the significant increased paw volume and paw thickness in arthritis control (table 1 and 2). Rasa Mezhugu $500 \mathrm{mg} / \mathrm{kg}$ on $30^{\text {th }}$ day showed a decrease in paw thickness was the evident significant prevention of paw edema (fig. 1,2 and 3).

\section{Histological findings}

Compared to normal control, the Freund's adjuvant induced arthritic control group showed the following changes in heamtoxylin and Eosin stained slides. The presence of the proliferation of synovial membrane, infiltration of the synovial membrane into the synovial cavity, accumulation of inflammatory cells adjacent to the inflamed joint and replacement of articular cartilage by inflammatory cells. The slides from collagen-induced arthritic rats presented partial destruction of the outer surface of the articular cartilages of the ankle joint with the other arthritic features which were present in the Freund's adjuvant-induced arthritic rats. These arthritic changes were absent in the Rasa Mezhugu treated rats with the dosage of $500 \mathrm{mg} / \mathrm{kg}$ treated rats $[12,13]$.

Histopathology slides of Ankle joint of Rasa Mezhugu treated rats showed normal synovial membrane, the anterior articular region with articular cartilage by the presence of no flocculation on the articular surface normal cellular organization; and the articular cavity has no synovial infiltration and the formation of pannus [14, 16]. The pictures show nearly normal histological features with almost intact bone and cartilage, in addition to normal joint space (fig. 4-7).

The bone marrow of Rasa Mezhugu treated rats (high/low/Middle dose) found within the central cavities of with hematopoietic tissue islands and adipose cells surrounded by vascular sinuses interspersed within a meshwork of bone (fig. 8-10). The cerebrum showed the absence of neuronal degeneration, edema and gliosis (fig. 11-13). The Cardiac muscle revealed normal appearances of the centrally arranged nucleus, connective tissue with distinct cardiac muscle fibers were well arranged (fig. 14-16). The kidney shows no glomerular or vascular injury the bowman's capsule with distinct polymorphonuclear cell nuclei with normal cells of renal cortex (fig. 17-19). The liver cells showed the normal microscopic architecture of the liver with hexagonal lobules and acini with the distinct central vein, portal triad, portal vein and hepatic artery with parenchyma cells (fig. 20-22). The lungs photos revealed that normal cytoarchitectural with predominant smooth muscle layer of the bronchi with enlargement of the alveoli and the alveoli sacs (fig. 23-
25). The pancreas with normal acinar cells arranged in lobules with prominent nuclei. The islet cells are embedded within the acinar cells and surrounded by a fine capsule with normal histological structure (fig. 26-28). The spleen showed no changes congestion, hemorrhage, edema, and erosions the cells are a clear and proper arrangement (fig. 29-31). Photomicrograph of stomach mucosal layer has shown normal cells with distinct mucosa, submucosa, muscle layers and serosal layers showing normal architecture (fig. 32-34). The tests of rat's revealed normal architecture with normal germinal epithelium, fully mature sperms filled the lumen of seminiferous tubules with tubular epithelium cells, absences of vascular congestion/interstitial edema and degenerated cells (fig. 35-37).

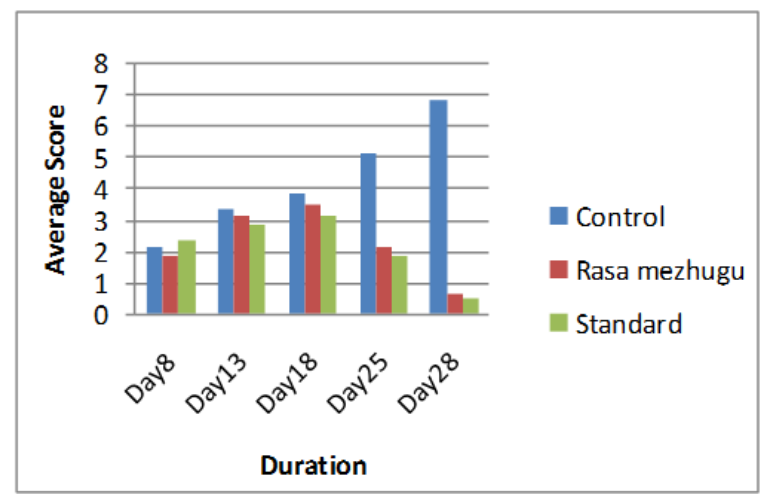

Fig. 1 Arthritic index

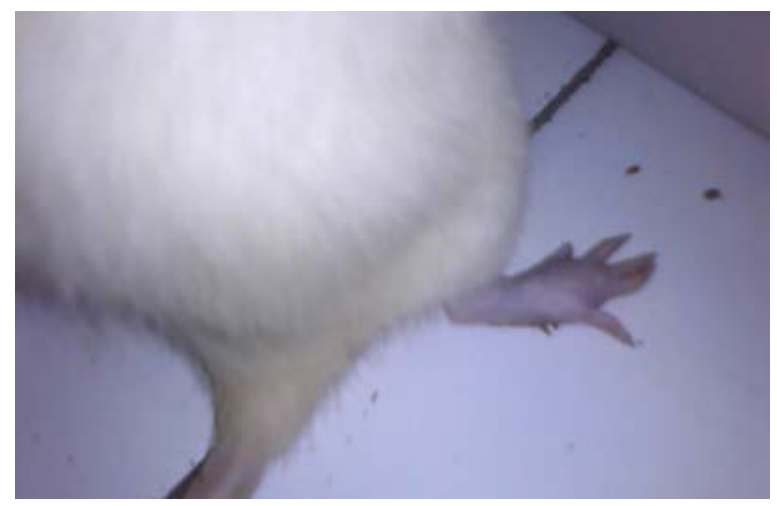

Fig. 2 Arthritic induced Right Palm

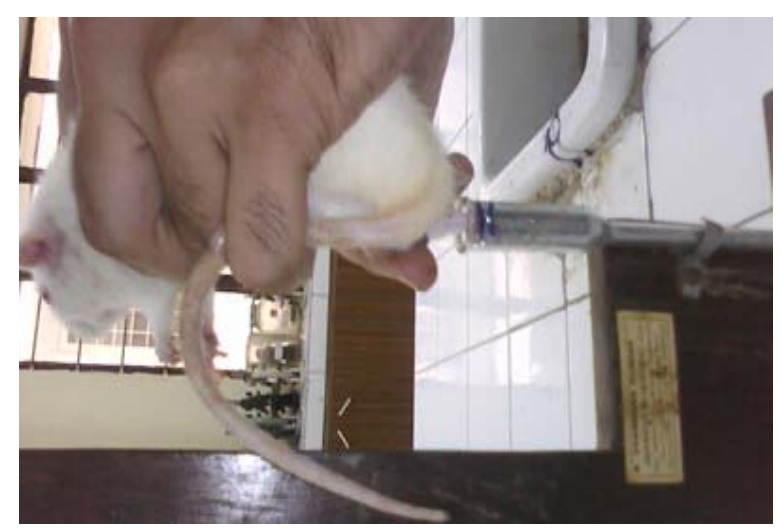

Fig. 3 Paw volumes measuring in mercury 


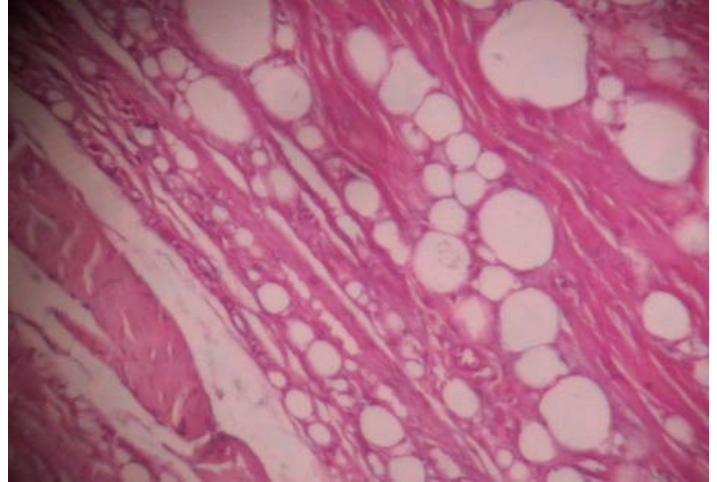

Fig. 6: Histopathology slides-Ankle joint of the Indomethacin treated animals

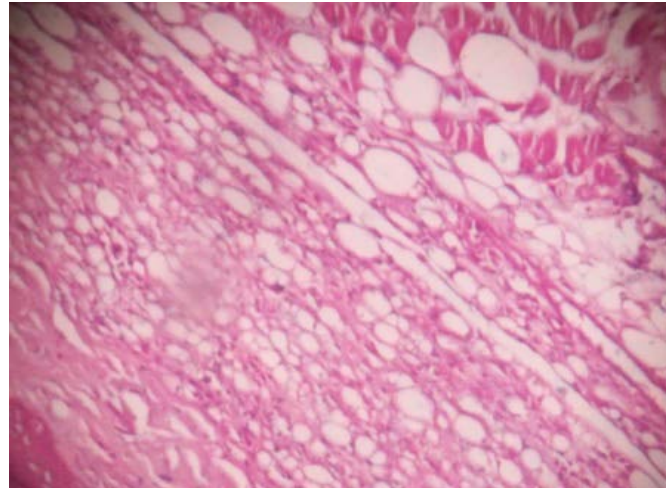

Fig. 7: Histopathology slides-Ankle joint of the Rasa Mezhugu treated animals

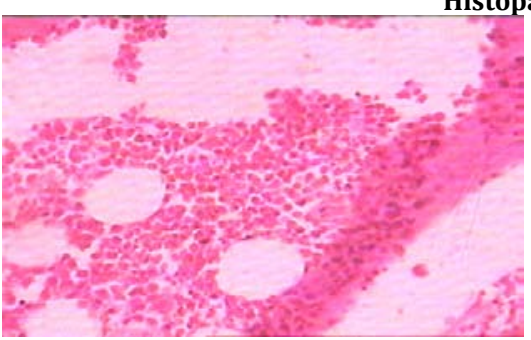

Fig. 8: Histopathology slide photos of Rasa Mezhugu-High dose treated animals' bone

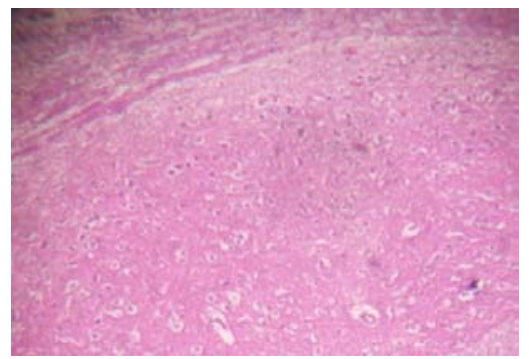

Fig. 11: Histopathology slide photos of Rasa Mezhugu-High dose treated animals' Brain

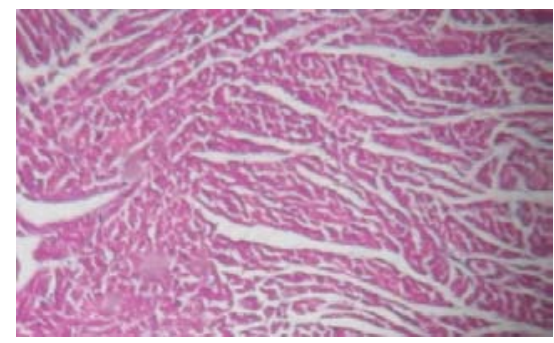

Fig. 14: Histopathology slide photos of Rasa Mezhugu-High dose treated animals' heart

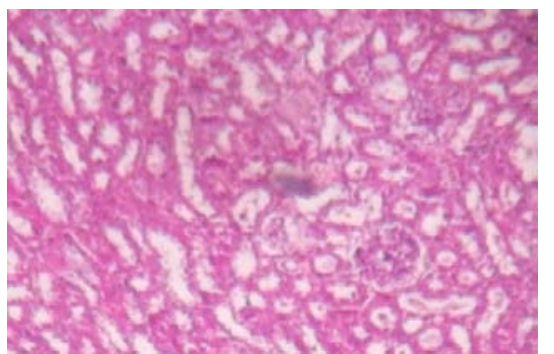

Fig. 17: Histopathology slide photos of Rasa Mezhugu-High dose treated animals' kidney

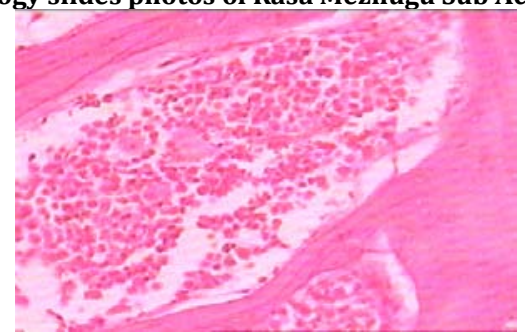

Fig. 9: Histopathology slide photos of Rasa Mezhugu-Low dose treated animals' bone

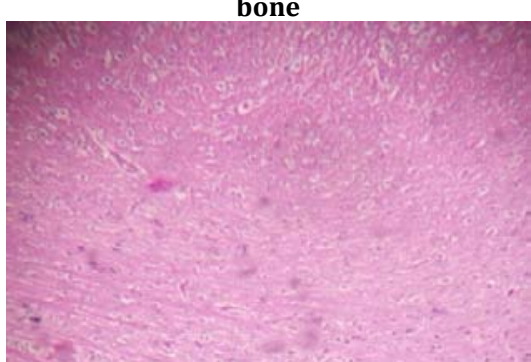

Fig. 12: Histopathology slide photos of Rasa Mezhugu-Low dose treated animals' Brain

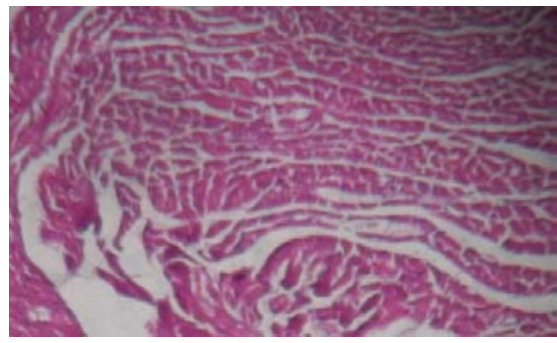

Fig. 15: Histopathology slide photos of Rasa Mezhugu-Low dose treated animals' heart

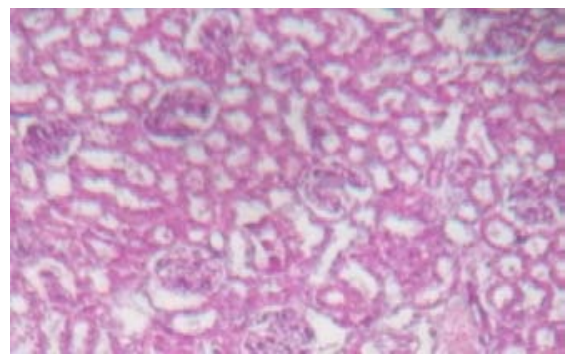

Fig. 18: Histopathology slide photos of Rasa Mezhugu-low dose treated animals' kidney

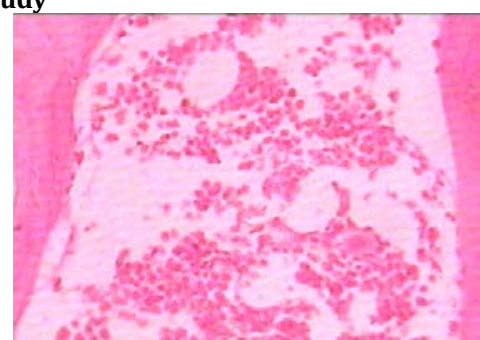

Fig. 10: Histopathology slide photos of Rasa Mezhugu-Middle dose treated animals' bone

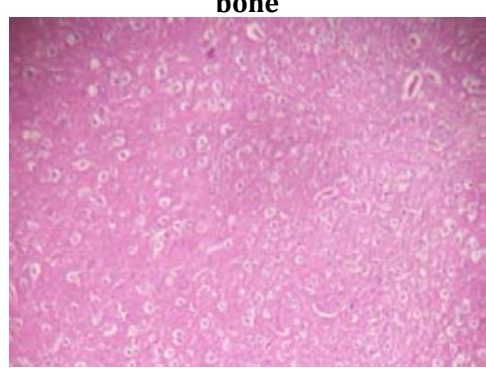

Fig. 13: Histopathology slide photos of Rasa Mezhugu-Middle dose treated animals' Brain

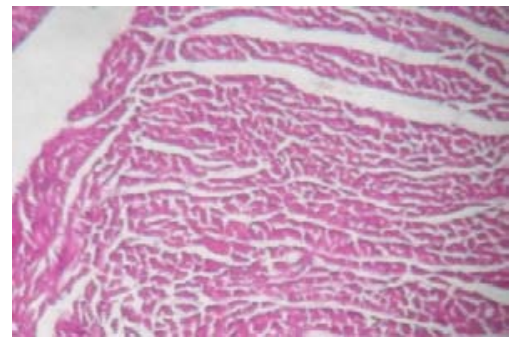

Fig. 16: Histopathology slide photos of Rasa Mezhugu-Middle dose treated animals' heart

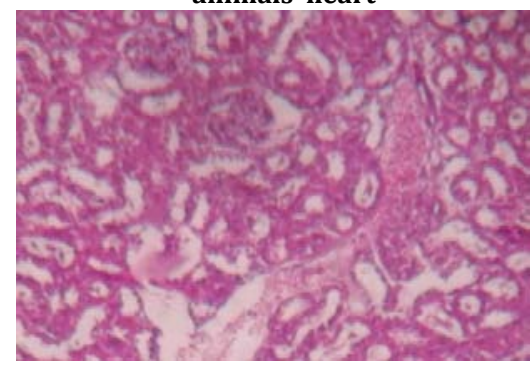

Fig. 19: Histopathology slide photos of Rasa Mezhugu-Middle dose treated animals' kidney 


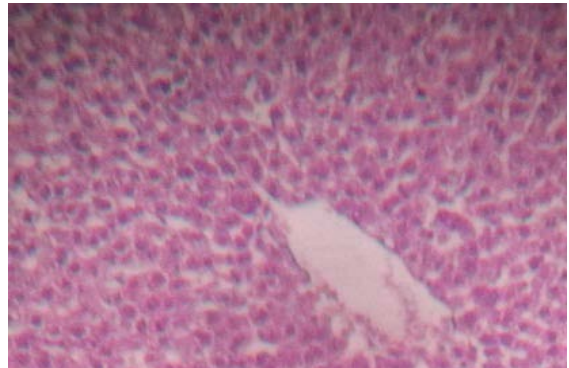

Fig. 20: Histopathology slide photos of Rasa Mezhugu-High dose treated animals' Liver

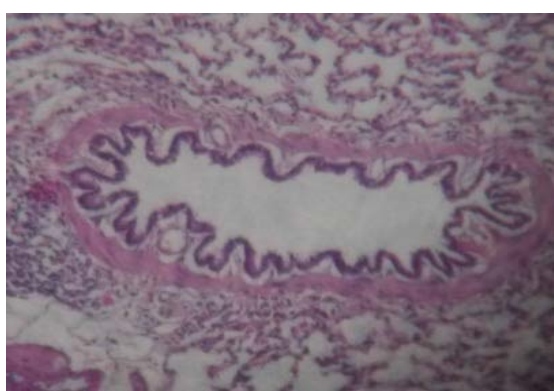

Fig. 23: Histopathology slide photos of Rasa Mezhugu-High dose treated animals'

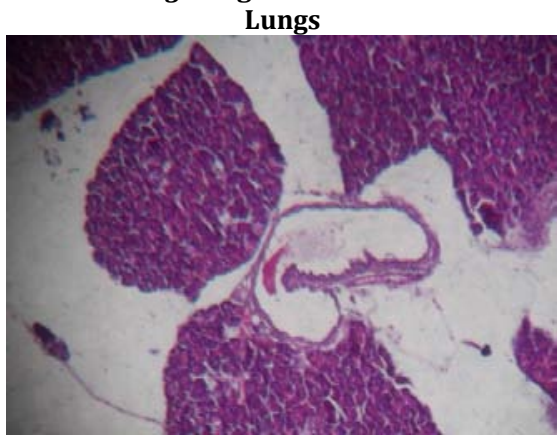

Fig. 26: Histopathology slide photos of Rasa Mezhugu-High dose treated animals

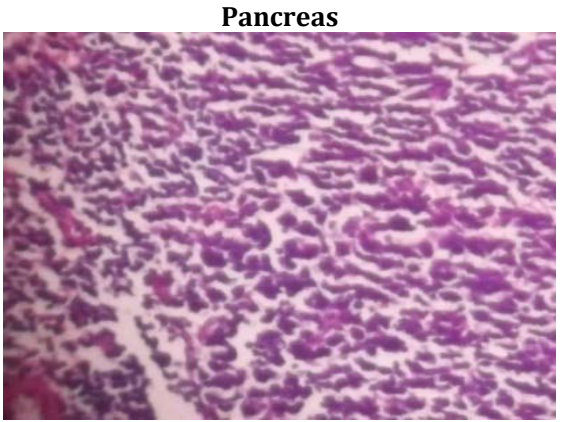

Fig. 29: Histopathology slide photos of Rasa Mezhugu-High dose treated animals'

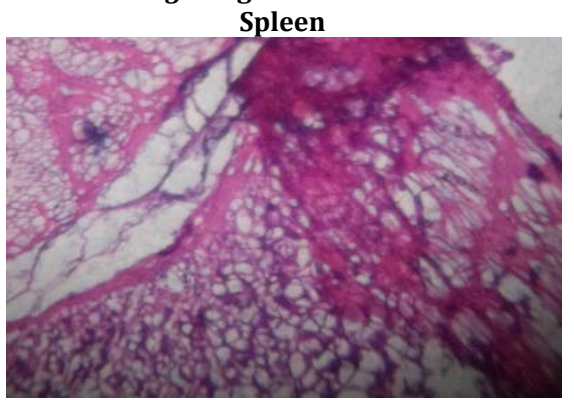

Fig. 32: Histopathology slide photos of Rasa Mezhugu-High dose treated animals' Stomach

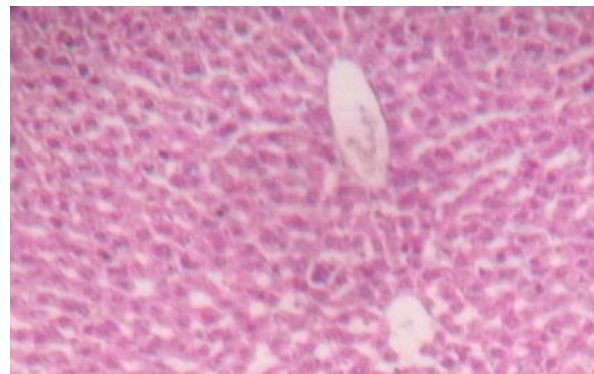

Fig. 21: Histopathology slide photos of Rasa Mezhugu-Low dose treated animals' Liver

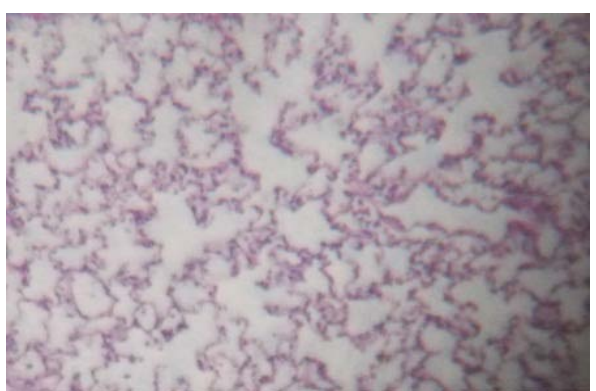

Fig. 24: Histopathology slide photos of Rasa Mezhugu-Low dose treated animals' Lungs

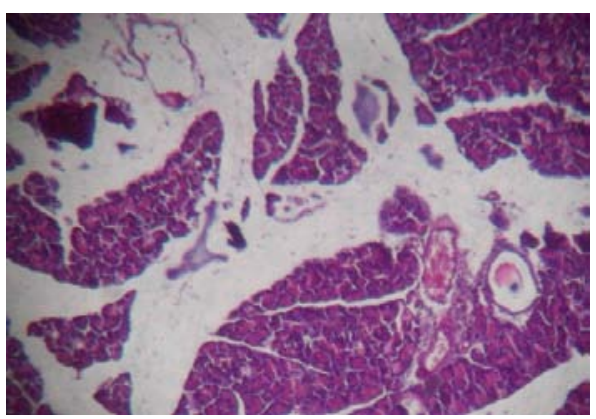

Fig. 27: Histopathology slide photos of Rasa Mezhugu-Low dose treated animals' Pancreas

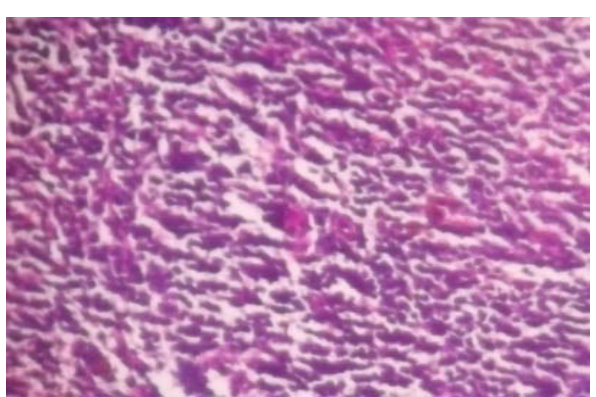

Fig. 30: Histopathology slide photos of Rasa Mezhugu-Low dose treated animals' Spleen

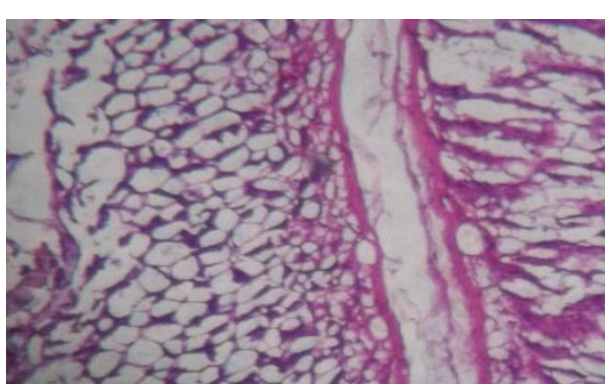

Fig. 33: Histopathology slide photos of Rasa Mezhugu-Low dose treated animals' Stomach

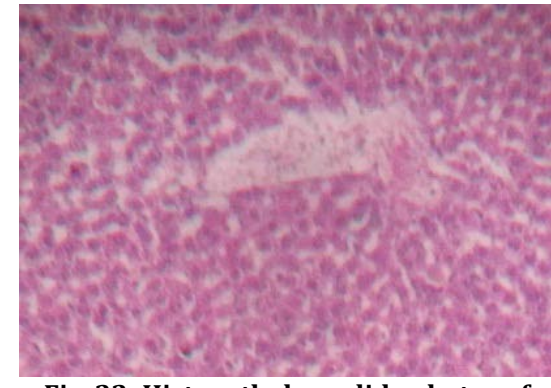

Fig. 22: Histopathology slide photos of Rasa Mezhugu-Middle dose treated animals' Liver

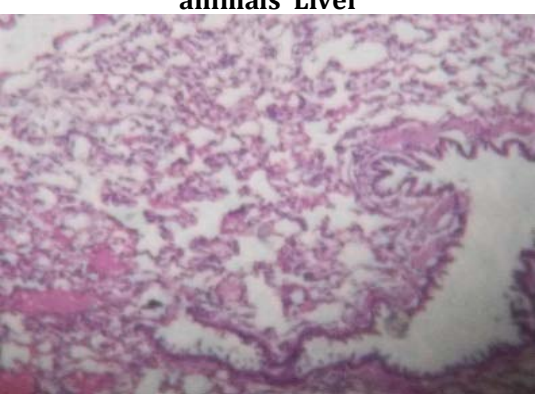

Fig. 25: Histopathology slide photos of Rasa Mezhugu-Middle dose treated animals' Lungs

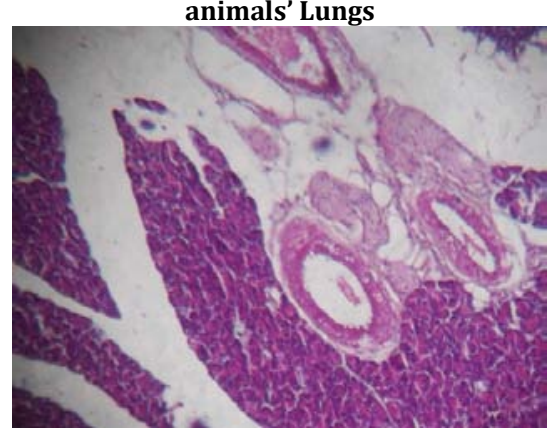

Fig. 28: Histopathology slide photos of Rasa Mezhugu-Middle dose treated animals' Pancreas

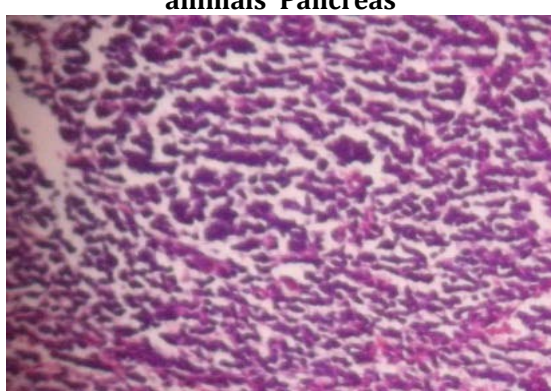

Fig. 31: Histopathology slide photos of Rasa Mezhugu-Middle dose treated animals' Spleen

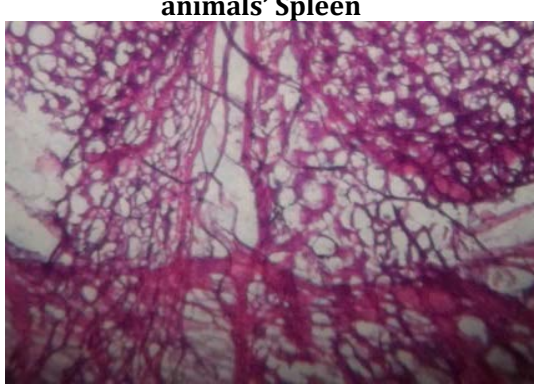

Fig. 34: Histopathology slide photos of Rasa Mezhugu-Middle dose treated animals' Stomach 


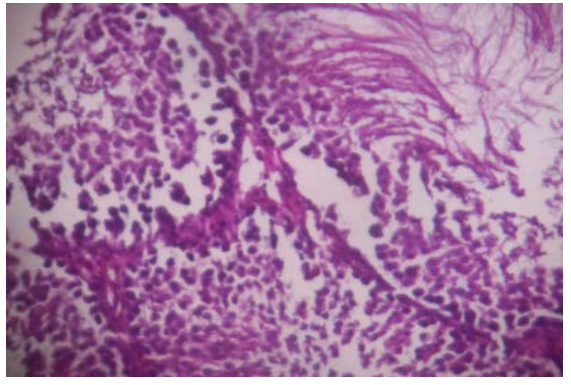

Fig. 35: Histopathology slide photos of Rasa Mezhugu-High dose treated animals' Testis

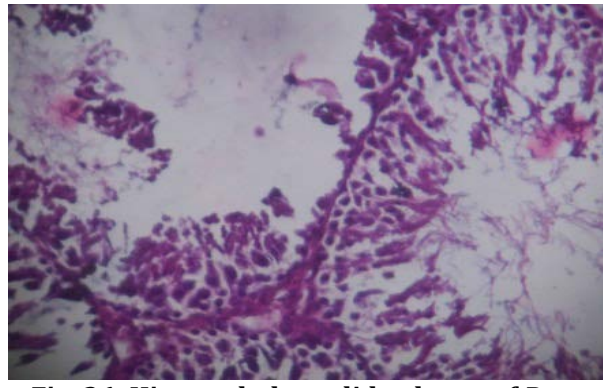

Fig. 36: Histopathology slide photos of Rasa Mezhugu-Low dose treated animals' Testis

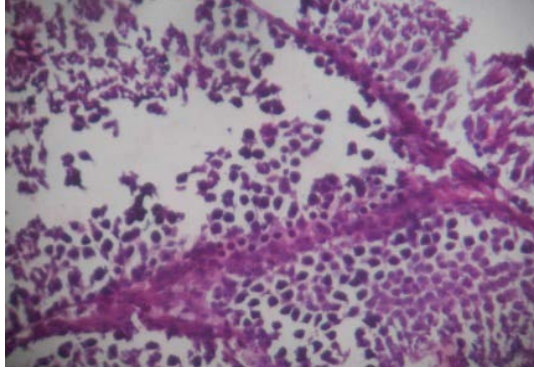

Fig. 37: Histopathology slide photos of

Rasa Mezhugu-Middle dose treated animals' Testis

Table 1: Effect of Rasa Mezhugu on paw volume in CFA induced arthritis in rats

\begin{tabular}{|c|c|c|c|c|c|c|c|}
\hline \multirow[t]{2}{*}{ Group } & \multirow[t]{2}{*}{ Treatment } & \multirow[t]{2}{*}{ Dose } & \multicolumn{5}{|c|}{ \% Inhibition of paw edema } \\
\hline & & & $8^{\text {th }}$ Day & $13^{\text {th }}$ day & $18^{\text {th }}$ day & $25^{\text {th }}$ day & $28^{\text {th }}$ day \\
\hline Normal & Saline & $2 \mathrm{ml} / \mathrm{kg}$ & -- & -- & -- & -- & -- \\
\hline Control (RA) & Palm Jaggary & $2 \mathrm{ml} / \mathrm{kg}$ & -- & -- & -- & -- & -- \\
\hline Test & Rasa Mezhugu & $500 \mathrm{mg} / \mathrm{kg}$ & -- & 17.33 & 40.19 & 62.10 & 78.29 \\
\hline Standard & Indomethacin & $10 \mathrm{mg} / \mathrm{kg}$ & -- & 19.76 & 44.81 & 68.35 & 89.46 \\
\hline
\end{tabular}

Table 2: Effect of Rasa Mezhugu on Arthritic Index (the secondary lesions score) in CFA induced arthritis in rats

\begin{tabular}{|c|c|c|c|c|c|c|c|}
\hline \multirow[t]{2}{*}{ Group } & \multirow[t]{2}{*}{ Treatment } & \multirow[t]{2}{*}{ Dose } & \multicolumn{5}{|c|}{ Arthritic index score } \\
\hline & & & 8 $^{\text {th }}$ Day & $13^{\text {th }}$ day & $18^{\text {th }}$ day & $25^{\text {th }}$ day & $28^{\text {th }}$ day \\
\hline Normal & Saline & $2 \mathrm{ml} / \mathrm{kg}$ & -- & -- & -- & -- & -- \\
\hline Control (RA) & Palm Jaggary & $2 \mathrm{ml} / \mathrm{kg}$ & 2.16 & 3.33 & 3.83 & 5.16 & 6.83 \\
\hline Test & Rasa Mezhugu & $500 \mathrm{mg} / \mathrm{kg}$ & 1.83 & 3.16 & 3.52 & 2.16 & 0.66 \\
\hline Standard & Indomethacin & $10 \mathrm{mg} / \mathrm{kg}$ & 2.33 & 2.83 & 3.16 & 1.83 & 0.5 \\
\hline
\end{tabular}

\section{CONCLUSION}

In adjuvant-induced arthritis model, rats developed a chronic swelling in multiple joints with the influence of inflammatory cells, erosion of joint cartilage and bone destruction and remodeling. Paw volume significantly increased on the $8^{\text {th }} 13^{\text {th }}$ and $18^{\text {th }}$ days after CFA injection compared to day 0 . But in the group treated with Rasa Mezhugu shows paw volume was gradually increased on the $18^{\text {th }}$ day compared to the $8^{\text {th }}$ day but on the $28^{\text {th }}$ day it is significantly reduced after CFA injection $(\mathrm{P}<0.05$, respectively). Additionally, there were no significant differences in paw volume between CFAinjected rats and rats injected with CFA and treated with adjuvant Palm jaggery.

\section{CONFLICTS OF INTERESTS}

\section{Declared none}

\section{REFERENCES}

1. Henderson B, Pettipher ER, Higgs GA. Mediators of rheumatoid arthritis. Br Med Bull 1987;42:415-28.

2. Findlay DM, DR Haynes. Mechanisms of bone loss in rheumatoid arthritis. Mod Rheumatol 2005;15:232-40.

3. Atzeni F, P Sarzi-Puttini. Early rheumatoid arthritis. Reumatismo 2007;59:100-17.

4. Fairhurst AM, PK Wallace, AS Jawad, NJ Goulding. Rheumatoid peripheral blood phagocytes are primed for activation but have impaired Fc-mediated generation of reactive oxygen species. Arthritis Res Ther 2007;9:R29-R29.

5. Hazeena BV, Sadique J. Long-term effect of herbal drug Withania somnifera on adjuvant-induced arthritis in rats. Indian J Exp Biol 1988;26:877-82.

6. Hoffmann JC, C Herklotz, H Zeidler, B Bayer, H Rosenthal, J Westermann. Initiation and perpetuation of rat adjuvant arthritis is inhibited by the anti-CD2 monoclonal antibody (mAb) OX34. Ann Rheum Dis 1997;56:716-22.
7. Fereidoni M, An Ahmadiani, S Semnanian, M Javan. An accurate and simple method for measurement of paw edema. J Pharmacol Toxicol Methods 2000;43:11-4.

8. OECD Guideline for the testing of chemicals, Acute Oral Toxicity: Up-and-Down Procedure 425; 1998. p. 1-8.

9. OECD Guidelines for the testing of chemicals. Repeated Dose 28-Day oral toxicity study in rodents 407; 2008. p. 1-13.

10. Ghosh MN. Fundamentals of experimental pharmacology. 2nd Edn. Scientific book agency, Kolkatta; 1994. p. 153-8.

11. Hurley JV, DA Willoughby. Acute inflammation: a combined topographical and electron microscopic study of the mode of action of carrageenan. Pathology 1973;5:9-21.

12. The disease-modifying activity of SK and F 106615 in rat adjuvant-induced arthritis. Multiparameter analysis of disease magnetic resonance imaging and bone mineral density measurements. Arthritis Rheum 1996;39:504-14.

13. Chen XY, Li J, Cheng WM, Jiang H, Xie XF, Hu R. Effect of total flavonoids of Chrysanthemum Indicum on the apoptosis of synoviocytes in joint of adjuvant arthritis rats. Am J Chin Med 2008;36:695-704.

14. Abotsi WMK, E Woode, GK Ainooson, An Amo-Barimah, E Boakye-Gyasi. Antiarthritic and antioxidant effects of the leaf extract of ficus exasperata P. Beauv. (Moraceae). Pharmacogn Res 2010;2:89-97.

15. Agarwal SK, MB Brenner. The role of adhesion molecules in synovial inflammation. Curr Opin Rheumatol 2006;18:268-76.

16. Akaogi J, T Nozaki, M Satoh, H Yamada. The role of PGE 2 and EP receptors in the pathogenesis of rheumatoid arthritis and as a novel therapeutic strategy. Endocr Metab Immune Disord Drug Targets 2006;6:383-94.

\section{How to cite this article}

- $\quad$ K Nandhagopal, M Kanniyakumari. Effect of rasa mezhugu on freund's adjuvant-induced arthritis in rats. Int J Curr Pharm Res 2016;8(4):80-85. 